
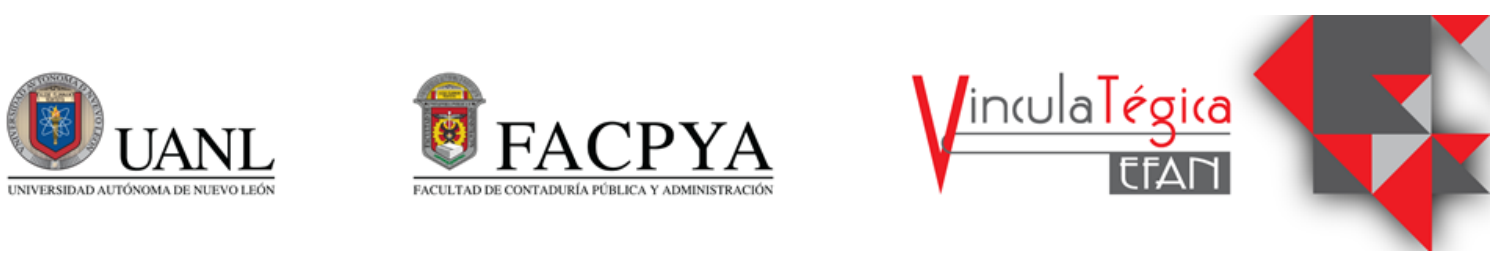

\title{
Factores que influyen en la adopción del e-commerce: un caso de estudio para los micronegocios del Área Metropolitana de Monterrey frente a la crisis sanitaria
}

\author{
José Manuel Manzanárez Pérez ${ }^{1}$, Jesús Fabián López Pérez² y Jesús Gerardo Cruz Álvarez ${ }^{3}$ \\ ${ }^{1}$ Estudiante del Doctorado en Filosofía con especialidad en administración de la Facultad de Contaduría \\ Pública y Administración de la Universidad Autónoma de Nuevo León, jmanzanarezpe@uanl.edu.mx, Pedro \\ de Alba S/N, Niños Héroes, Ciudad Universitaria, San Nicolás de los Garza, N.L. \\ ${ }^{2}$ Profesor-Investigador de la Facultad de Contaduría Pública y Administración de la Universidad Autónoma \\ de Nuevo León, fabian.lopez@gmail.com, Pedro de Alba S/N, Niños Héroes, Ciudad Universitaria, San \\ Nicolás de los Garza, N.L. \\ ${ }^{3}$ Profesor-Investigador de la Facultad de Contaduría Pública y Administración de la Universidad Autónoma \\ de Nuevo León, jesusphd@prodigy.net.mx, Pedro de Alba S/N, Niños Héroes, Ciudad Universitaria, San \\ Nicolás de los Garza, N.L.
}

Información del artículo revisado por pares
Fecha de aceptación: junio-2021
Fecha de publicación en línea: diciembre-2021
DOI: https://doi.org/10.29105/vtga7.1-85

\section{Resumen}

El objetivo de este artículo es analizar la adopción del comercio electrónico en microempresas, de venta minorista y de servicios del Área Metropolitana de Monterrey (AMM) ante la crisis sanitaria. Basado en el modelo en la conducta de aceptación tecnológica de los microempresarios. Los resultados proporcionaron evidenciar la adopción del comercio electrónico en AMM, por la fuerte relación entre las diferentes características personales y descriptivas de los microempresarios.

Palabras clave: comercio electrónico, crisis sanitaria, micronegocios.

\section{INTRODUCCIÓN}

Las Microempresas figuran con más del $95 \%$ del total de las empresas y entre el $60 \%$ y el $70 \%$ del empleo y generan una gran proporción de nuevos puestos de trabajo en las economías de acuerdo con la Organización para la Cooperación y el Desarrollo Económicos (OCDE, 2000). Tienen sus

\begin{abstract}
The objective of this article is to analyze the adoption of electronic commerce of the microenterprises, commercial, and service businesses in the Monterrey Metropolitan Area (AMM) in the face of the health crisis. Based on the Technological acceptance model of microentrepreneurs. The results provided evidence of the adoption of electronic commerce in AMM, due to the strong relationship between personal and descriptive characteristics of the microentrepreneurs.
\end{abstract}

Keywords: e-commerce, sanitary crisis, microbusiness.

JEL: C01, L81, L26.

fortalezas y oportunidades específicas que pueden requerir replicas políticas especiales. Los micronegocios necesitan perfeccionar sus habilidades de gestión, su capacidad para recopilar datos y su experiencia tecnológica.

Conforme a los datos de la Organización de las Naciones Unidas para la Educación, la Ciencia y la Cultura (UNESCO, 
2020) 188 países incluido México han implementado el cierre de universidades, sitios de culto, negocios y empresas no esenciales como respuesta ante el emplazamiento generado por la epidemia, lo cual significa que más del $70 \%$ de las personas en el planeta se encuentra en confinamiento. La crisis ha propiciado el cierre de casi 10 mil empresas en el país en los primeros meses del inicio de la pandemia, la mayoría micro y pequeñas.

Ante esta problemática, la respuesta de las autoridades a la situación ocasionada por la crisis sanitaria SARS-CoV-2, mejor conocida como COVID- 19 ha sido un par de acciones de ayuda a los microempresarios de México durante la epidemia, han sido los créditos directos como apoyo del presidente de la república, de aproximadamente $\$ 1,000$ (mil dólares), fue un ayuda expedita en un breve plazo para el apoyo en tanto el cierre de operaciones comerciales durante el confinamiento que se otorgó a 1.5 millones de microempresarios y el dote de instrumentos para incrementar la eficacia, declaró la Secretaria de Economía, en apoyo con Banco de Desarrollo de América Latina (Forbes, 2020). Se estaba consciente desde el comienzo del encierro se sabía que tendría una importante afectación en los micronegocios y particularmente en los ámbitos más sensibles reconocidos. Para eso se elaboró un programa para otorgar apoyos directos a las personas y negocios más frágiles, "en este caso a los micronegocios", indicó la Secretaría de Economía (SE, 2020).

Para el caso específico de los microempresarios del Área Metropolitana de Monterrey (AMM) de Nuevo León, cabe preguntar ¿realmente estos empresarios están dispuestos a adoptar una plataforma de $e$ commerce; y ¿cuáles son los factores que influyen en la adopción del e-commerce en los micronegocios, para establecer su plataforma de comercio electrónico. en estos tiempos de la crisis sanitaria. A partir de estas interrogantes, la presente investigación analiza los factores que influyen en la adopción que incurren los micronegocios del AMM para fundar el modelo de negocio en e-commerce en medio de la situación que enfrenta la sociedad por la crisis sanitaria.

Para lograr el objetivo de la investigación y responder a las preguntas planteadas, se implementa un cuestionario en línea a los microempresarios registrados en la incubadora de negocios de la Facultad de Contaduría Pública y Administración. Para ello se aplicará un modelo Likert de siete elementos, que permitirá explicar la relación existente entre la adopción en adquisición de una plataforma digital y de esa manera aplicar cualquier modalidad del e-commerce y las características socioeconómicas, estructurales, sociales y geográficas de los micronegocios.

\section{MARCO TEÓRICO}

\subsection{Tipos, clasificación y usos del $e$ - commerce en el mundo}

De acuerdo con Eduardo Líberos e Ignacio Somalo (2011) los tipos de comercio electrónico de acuerdo con el modelo o rol que se participa en las transacciones puede ser:

- Empresa-Empresa: B2B (business to business): interacción electrónica entre dos negocios. También conocido como negocio mayorista o de distribuidores que realizan transacciones comerciales a través de Internet $y$ en el que participan empresas o corporaciones. Un ejemplo de este tipo de $e$ commerce se destaca entre los punteros a nivel global: Alibaba al realizar un pedido electrónico, donde alguien juega el rol de vendedor y otro de comprador. El B2B describe las operaciones comerciales entre negocios, entre un productor $\mathrm{y}$ un distribuidor, o entre un mayorista y un minorista. El comercio electrónico puede ahorrarle o hacer que las empresas tengas mejores utilidades.

El B2B se contextualiza en el ámbito de la comunicación y la colaboración. Bastantes empresas ahora utilizan las redes sociales para contactar directamente a sus proveedores; sin embargo, ahora están usando instrumentos tecnológicos dentro de la empresa para apoyar

\footnotetext{
${ }^{1}$ El e-commerce es el uso de plataformas electrónicas como, por ejemplo: email, sitios web, intercambio electrónico de datos (EDI), y protocolo de transferencia de datos (FTP).
} 
a los empleados puedan vincularse con los fabricantes. Cuando el intercambio tiene lugar entre los trabajadores, esto se puede denominarse interacción B2B. Se está incrementando el uso en muchas organizaciones implementando el comercio electrónico B2B en numerosas formas y recibiendo beneficios tangibles. Pero como el comercio electrónico madura y se desarrolla, estas formas son es probable que cambie en función de la tasa de adopción acelerada. Según la revisión, tres modelos están comenzando a aparecer en el mercado.

Basado en transacciones: Una sola empresa establece un método transaccional común para realizar negocios con sus principales clientes o proveedores clave. Esta oferta es común en todas las unidades de negocio dentro del empresa e incluye herramientas, técnicas e infraestructura.

Basado en procesos: Dos empresas establecen un proceso comercial común para realizar negocios de manera eficiente $y$ efectiva entre las dos empresas. Las dos firmas establecen y comparten prácticas comunes de manera conjunta, tanto dentro de su firma como fuera de su organización con este comercio predeterminado.

Basado en relaciones estratégicas: Dos o empresas que establecen una asociación de relación estratégica basado en todas las interacciones importantes entre las organizaciones.

Esto incluye transacciones, procesos y cualquiera otra colaboración entre las dos organizaciones. De perspectiva tecnológica esto incluye vincular el CRM, ERP y sistema SCM de las dos organizaciones. De esta manera cada organización puede monitorear la actividad de ventas, producción horarios, gestión de inventarios y servicios técnicos intercambiar. Algunas formas en que las empresas se han beneficiado del comercio electrónico B2B son:

- Gestionar el inventario de forma más eficiente.

- Ajustando más rápidamente las demandas de los clientes.

- Llevar los productos al mercado más rápido.
- Reducir el costo del papeleo (paperless).

- Redefiniendo el proceso de compras.

- Obtener precios más bajos en algunos suministros

- Empresa-Consumidor: B2C (business to consumer): Comercio entre empresas y consumidores finales. Se le conoce como comercio minorista o retail, ejemplo Amazon.com, es la traslación directa del modelo de negocio tradicional del comercio minorista al entorno digital. B2C consiste en varios elementos como clasificación (catálogos), pedido, planificación, generación, estimación de costos, precios, orden, tiempo de entrega, mantenimiento de productos, categoría gestión, pedido y procesamiento de tarjetas de crédito.

Catálogos: es el proceso de mostrar elementos de una base de datos basada en categorías y subcategorías seleccionadas por los clientes. Crea un muy simple sistema de comercio electrónico que permite a los espectadores seleccionar elementos para comprar en el catálogo de productos. Catalogar es una de las principales operaciones utilizadas en el comercio electrónico de empresa a consumidor. Los clientes pueden comunicarse con las líneas de la tienda a través del sistema de red utilizado en el negocio. Para este propósito, se debe requerir una técnica para cumplir con todas las necesidades del consumidor. Para hacer compras en línea, es necesario un catálogo, que describirá la calidad, precio y marca del artículo.

Planificación de órdenes y generación de pedidos: La planificación y generación de pedidos permite el inicio de pedidos individuales, así como pedidos en bloque de una manera fácil de usar y flexible. Cada pedido pasa por un ciclo de vida completo. El estado de un pedido indica en qué etapa del ciclo de vida de una orden, es decir, inicia valida, validado con aviso, enviado o cancelado.

- Empresa-Administración: $\quad B 2 A$ (Business to Administration). Es muy reconocida igualmente como empresagobierno, cubre los nexos entre las entidades empresa y organizaciones de gobierno. El 
exponente B2A consta de una serie de operaciones o transacciones que se obtienen entre las empresas y las entidades gubernamentales, la autoridad típicamente ofrece la asistencia a las organizaciones, son el enlace entre lo pedagógico o recaudación fiscal, afín al empleo y la asistencia social, salud pública o privada, etc. Las estructuras de beneficios de servicios suelen ser tramitadas por la misma entidad, así como los sistemas de paga. Esta clasificación o uso del e-commerce: empresa-administración no puede considerarse del todo una articulación comercial, en realidad sí se pueden generar pagos derivados de contribuciones, tasa de interés etc.

Algunas de las utilidades procedente de este esquema Empresa-Administración, está la posibilidad de utilizar cuestionarios administrativos para entrada practica y sencilla a trámites de envío y recepción de datos administrativos, actualización de la información, reducción de gastos en los trámites burocráticos, una reducción significativa de tiempo en la tramitación de servicios administrativos, entre otros beneficios.

- Consumidor-Consumidor: $\mathrm{C2C}$

(Customer to Customer) Los consumidores compran y venden entre ellos a través de una plataforma digital (Liberos y Somalo, 2011).

Similar a las transacciones B2A, en este proceso las interacciones con la administración se realizan entre consumidores finales o individuales, en lugar de negocios y gobierno. La definición abarca todas las compraventas digitales se llevan a cabo entre usuarios particulares y las instituciones públicas, algunos pueden ser, servicios como por ejemplo agendar cita médica o para prestaciones sociales a través de plataformas electrónicas en internet, los tramites de impuestos o seguridad social en línea, etc., el objetivo es que todos los tramites con la administración sean más prácticas, sencillas y accesibles para todos.

El modelo $\mathrm{C} 2 \mathrm{C}$ o Consumer to consumer deduce la existencia de una forma de adquirir $\mathrm{y}$ vender entre consumidores individuales que emplean algunas plataformas electrónicas como por ejemplo ebay, en su mayoría surgieron de las nuevas tecnologías de la información y la veloz expansión del Internet. De estos casos los consumidores realizan transacciones de compra y venta entre consumidores finales y sin que las empresas propietarias de los bienes o servicios participen directamente. Algunas de las prerrogativas de $\mathrm{C} 2 \mathrm{C}$ son:

- Hace accesible la comercialización, adquisición y venta entre personas.

- Permite entrar en el mercado a prácticamente cualquier persona.

- $\quad$ Es posible la localización de bienes o servicios especiales o escasos.

- Es posible enlazar distintas ubicaciones geográficas.

El sistema $\mathrm{C} 2 \mathrm{C}$ se ha generalizado de forma gradual hasta la actualidad por el considerable baja de costos de los servicios del internet y la erradicación de un sin número de intermediarios en las operaciones electrónicas. Asimismo, la mejora en el desarrollo de los sistemas de pago por tarjetas de crédito o débito y los monederos electrónicos y la seguridad que ofrecen estos métodos ha dado confianza a los usuarios que cada vez más continuamente con mayor frecuencia son parte del dinamismo de la compra-venta a través de medios electrónicos en la red de internet.

\subsection{Situación actual del e-commerce en México}

En México, de acuerdo con los datos obtenidos de los últimos censos económicos del INEGI (2019), las unidades económicas totalizaron, a nivel nacional, 6269309 unidades, y de estas el $99.8 \%$ emplea a menos de 250 trabajadores. En ese sentido, existen alrededor de 6256770 micros y pequeñas empresas, y de estas, 5,955,844 son micronegocios que cuentan con menos de 10 trabajadores. Es decir que, del total de empresas del país, según el censo económico, el $95 \%$ se considera microempresa.

Como se muestra en la Tabla 2, el INEGI (2019) concluyó que se emplea a 35 463625 de personas; en ese contexto, la 
categoría más relevante es la que corresponde a la microempresa, pues esta aporta el $37.8 \%$ de personal ocupado.

Tabla 1. Características de establecimientos en México

\begin{tabular}{|c|c|c|c|}
\hline $\begin{array}{c}\text { Categoría de } \\
\text { establecimientos }\end{array}$ & Cantidad de personas & $\begin{array}{l}\text { Porcentaje de } \\
\text { establecimientos }\end{array}$ & $\begin{array}{l}\text { Cantidad de } \\
\text { establecimientos }\end{array}$ \\
\hline Micro & 0 a 10 personas. & $95.0 \%$ & $\approx 5955844$ \\
\hline Pequeños & 11 a 50 personas. & $4.0 \%$ & $\approx 250772$ \\
\hline Medianos & 51 a 250 personas. & $0.8 \%$ & $\approx 50154$ \\
\hline Grandes & Más de 250 personas. & $0.2 \%$ & $\approx 12539$ \\
\hline
\end{tabular}

Fuente: (INEGI, 2019)

En México, a nivel sectorial, la categoría de servicios es la más importante en cuanto a número de unidades económicas, dado que representa al $48.7 \%$ del total de unidades. Este dato supone que 3053153 de las unidades económicas del país se desempeñan en actividades de servicios.

Tabla 2. Personal ocupado en México por categoría de establecimiento

\begin{tabular}{ccc}
\hline Categoría de establecimientos & Cantidad de personas ocupadas & $\begin{array}{c}\text { Porcentaje de personal ocupado a } \\
\text { nivel nacional }\end{array}$ \\
\hline Micro & 0 a 10 personas. & $37.8 \%$ \\
Pequeños & 11 a 50 personas. & $14.7 \%$ \\
Medianos & 51 a 250 personas. & $15.9 \%$ \\
Grandes & Más de 250 personas. & $31.6 \%$
\end{tabular}

Fuente: Elaboración propia.

Tabla 3. Revisión de la literatura sobre la adopción del e-commerce.

\begin{tabular}{|c|c|c|}
\hline Autores & País & Hallazgos \\
\hline $\begin{array}{l}\text { Londoño y Tavera } \\
\text { (2014) }\end{array}$ & Colombia & $\begin{array}{l}\text { Los autores proponen un modelo teórico que compone en su, } \\
\text { junto los constructos de seguridad, confianza e "innovativeness", } \\
\text { para aclarar elfenómeno de la adopción tecnológica del e-commerce } \\
\text { en países emergentes. }\end{array}$ \\
\hline
\end{tabular}


Jones, Alderete y Motta

(2013)

Argentina

Luen, Chan, y Parker (2004)

Australia

Kang y Ick $(2015$

Yaaghoob, Jafarpour y Esmaeilpour (2016)

Irán

Mutum y Ghazali

(2006)

Malasia

Su investigación de empresas en Brunéi para identificar relaciones entre las percepciones de los gerentes sobre el valor estratégico del comercio electrónico y las variables que pueden influir en el comercio electrónico. Dos de cada tres factores de valor estratégico percibidos son significativos, mientras que cuatro de cada de cinco factores de adopción son un determinante significativo del comercio electrónico.

Seyal y Rahim (2010) Brunéi electrónico.

Fuente: Elaboración propia.

\section{MÉTODO}

\subsection{Descripción de los datos}

Se utilizaron los datos de la investigación "Determinantes del gasto en e-commerce debido al covid-19" (Alvarado, 2021). Se contó con la participación de microempresarios del área metropolitana de Monterrey. Los datos analizados son de corte transversal y se aplica una encuesta en línea en el periodo de marzo y junio de 2020 a través de la plataforma del OCDEM. Se contó con la participación de 661 microempresarios o personas autónomas que se registraron para tomar algún curso en línea o webinars, o que estaban inscritas en la incubadora de negocios de la Facultad de Contaduría Pública y Administración.

Con la información recabada se efectuó un modelo Likert de siete elementos, el cual se explicará más adelante en la sección de 
Método de análisis.

Para lograr el objetivo planteado en la presente investigación e identificar los factores que influyen en la adopción del $e$ commerce: un caso de estudio para los micronegocios del área metropolitana de monterrey frente a la crisis sanitaria. El gasto de los microempresarios para adquirir una plataforma de e-commerce, se analizaron algunas características estructurales y socioeconómicas de los micronegocios, tal como se observa en la Tabla 4.

Tabla 4. Descripción de las variables

\begin{tabular}{ll}
\hline Variables & Descripción \\
\hline Inversión & $\begin{array}{l}\text { Inversión que el microempresario realizó para la adquisición } \\
\text { de la plataforma de e-commerce en pesos mexicanos. }\end{array}$ \\
Edad & Edad del dueño o responsable del micronegocio. \\
Sexo & Variable dummy: $0=$ Mujer; 1 = Hombre. \\
Educación & Nivel de formación académica en años. \\
Antigüedad & Número de años que lleva activa el micronegocio. \\
Empleados & Número de trabajadores en el micronegocio \\
Clientes & Promedio mensual de clientes que frecuentan el micronegocio. \\
& Rendimiento promedio mensual del micronegocio en el último \\
Utilidad & año en pesos mexicanos.
\end{tabular}

Fuente: Elaboración propia.

El análisis descriptivo de las variables mencionadas se muestra en la Tabla 6, donde se aprecia que $35.5 \%$ de los microempresarios argumentó gastar o invertir en plataformas digitales para llevar a cabo las ventas de sus productos y servicios en la modalidad de $e$ commerce. Como resultado, el monto promedio fue de $5,100.44$ pesos, específicamente este gasto se dio en aquellos micronegocios que tienen más de dos años en operación (2.05). Asimismo, se aprecia que la edad promedio de los microempresarios participantes en este estudio fue de 44.92 años. Respecto a la muestra recabada, los microempresarios del sexo masculino fueron quienes más accedieron a contestar la encuesta
$(65.4 \%)$; la mayoría señaló que tiene a su cargo, en promedio, a dos personas.

\subsection{Método de análisis o modelo econométrico.}

Los datos analizados son de corte transversal que se tomó de la investigación "Determinantes del gasto en e-commerce debido al covid-19" (Alvarado, 2021).

La encuesta se aplicó en línea en el periodo de marzo y junio de 2020 a través de la plataforma del OCDEM. Se contó con la participación de 661 microempresarios o personas autónomas que se registraron para tomar algún curso en línea o webinars, o que 
estaban inscritas en la incubadora de negocios de la Facultad de Contaduría Pública y Administración. El tipo de muestreo que se realizó cuestionario a la población objetivo para la recolección de datos. Para ello se aplicará análisis estadísticos descriptivos, cubriendo el AMM del estado de Nuevo León, en forma general.

Tabla 5. Descripción de las variables

\begin{tabular}{ll}
\hline Variables & \multicolumn{1}{c}{ Descripción } \\
\hline Valor percibido & $\begin{array}{l}\text { Es la percepción del microempresario se encuentra satisfecho } \\
\text { por la adquisición de la plataforma de } e \text {-commerce. }\end{array}$ \\
$\begin{array}{l}\text { Calidad del } \\
\text { servicio }\end{array}$ & $\begin{array}{l}\text { Es la amplitud de la discrepancia o diferencia que exista entre } \\
\text { las expectativas o deseos de los clientes y sus percepciones. }\end{array}$ \\
Confianza en el e-commerce & $\begin{array}{l}\text { Se refiere a la seguridad establecida entre microempresario y el } \\
\text { consumidor haciendo negocios, las cuales, en el contexto del } \\
\text { comercio electrónico B2C (Business to Consumer }) \\
\text { corresponden al consumidor y al microempresario. }\end{array}$ \\
Satisfacción & Es actitud del microempresario hacia la plataforma $e$-commerce. \\
Adopción del e-commerce & $\begin{array}{l}\text { Es el comportamiento ante la intención de una conducta } \\
\text { mediante la actitud del microempresario, afectada por la utilidad } \\
\text { percibida y la facilidad de uso percibida. }\end{array}$ \\
\hline
\end{tabular}

Fuente: Elaboración propia.

Tabla 6. Estadísticos descriptivos de las variables

\begin{tabular}{ccccc}
\hline Variables & Mínimo & Máximo & Media & Desviación \\
\hline Inversión & 0 & 25326 & 5100.44 & 7434.85 \\
Edad & 30 & 60 & 44.92 & 8.08 \\
Sexo & 0 & 1 & 0.65 & 0.48 \\
Educación & 6 & 16 & 11.63 & 2.83 \\
Antigüedad & 1 & 8 & 2.15 & 1.12 \\
Empleados & 0 & 8 & 1.61 & 1.49 \\
Clientes & 18 & 112 & 63.77 & 26.48 \\
Utilidad & 750 & 48000 & 11637.90 & 6334.53 \\
\hline
\end{tabular}

Fuente: Elaboración propia.

\section{RESULTADOS}

En la Tabla 7 se presentan los coeficientes del modelo mínimo cuadrado ordinarios como variable dependiente se encuentra la inversión 
en e-commerce y como variables explicativas se encuentran la utilidad, escolaridad, género, empleados, edad y antigüedad. Se determinó que las variables socioeconómicas que forman parte de los factores de predisposición -en este caso, el número de empleados en el micronegocio y la edad del dueño - afectan la magnitud del gasto que se realiza para la compra de una plataforma de e-commerce, como se observa los modelos 1 y 3 . Además, se concluyó que cada año añadido en la edad representa una disminución de un $0.02 \%$ en el gasto de una plataforma de e-commerce y cada empleado adicional en el micronegocio disminuye la probabilidad de gasto en $0.22 \%$.

Tabla 7. Mínimos cuadrados ordinarios.

\begin{tabular}{|c|c|c|c|c|}
\hline Variable & Coeficiente & $\begin{array}{l}\text { Error } \\
\text { Estandar }\end{array}$ & $t$-Statistic & Prob. \\
\hline UTILIDAD & -0.042209 & 0.051325 & -0.822397 & 0.4112 \\
\hline SEXO & -1897.998 & 544.0057 & -3.48893 & 0.0005 \\
\hline ESCOLARIDAD & -2.757981 & 81.90755 & -0.033672 & 0.9732 \\
\hline EMPLEADOS & 269.6341 & 306.6686 & 0.879236 & 0.3796 \\
\hline EDAD & -92.05886 & 41.56158 & -2.214999 & 0.0271 \\
\hline CLIENTES & -31.46867 & 10.61954 & -2.963279 & 0.0032 \\
\hline ANTIGÜEDAD & -1002.383 & 347.7406 & -2.88256 & 0.0041 \\
\hline R-squared & 0.053198 & \multicolumn{2}{|c|}{ Mean dependent var } & 5100.44 \\
\hline Adjusted R-squared & 0.04204 & \multicolumn{2}{|c|}{ S.D. dependent var } & 7434.848 \\
\hline S.E. of regression & 7276.889 & \multicolumn{2}{|c|}{ Akaike info criterion } & 20.63599 \\
\hline Sum squared resid & $3.15 \mathrm{E}+10$ & \multicolumn{2}{|c|}{ Schwarz criterion } & 20.69447 \\
\hline Log likelihood & -6203.434 & \multicolumn{2}{|c|}{ Hannan-Quinn criter. } & 20.65875 \\
\hline F-statistic & 4.767855 & \multicolumn{2}{|c|}{ Durbin-Watson stat } & 0.108572 \\
\hline Prob(F-statistic) & 0.000031 & & & \\
\hline
\end{tabular}

Fuente: Elaboración propia.

\section{CONCLUSIONES}

El Modelo de Aceptación de Tecnología (Davis, 1989) resulta muy apropiado para explicar la adopción del comercio electrónico en el Área Metropolitana de Monterrey solamente se rechaza la relacionada con la facilidad percibida de uso sobre la actitud, tal como ocurre en otros estudios de aceptación de tecnologías digitales (Tavera y Lodoño, 2014)

En la presente investigación se evidenció que las peculiaridades específicas de los propietarios de los micronegocios las características descriptivas, predominan los del sexo masculino, de estos negocios se comprueban que tiene un impacto indudable y significativamente en la probabilidad de que los microempresarios inviertan en la adquisición y adopción de plataformas digitales en $e$-commerce de sus productos y servicios. Lo anterior pese a que el $64 \%$ de los microempresarios reportó no haber realizado ningún gasto al inicio de la pandemia para la modalidad de $e$-commerce en sus negocios. El resto de los microempresarios que si invirtieron $(36 \%)$ o adoptaron el e-commerce fue un monto relativamente bajo en promedio 5,100.00 pesos mexicanos.

De acuerdo con Molla (2004) y por igual con Tan et al. (2007) y Bravo (2011), los factores organizacionales de los micronegocios, así como las tecnologías de la información existentes, el esquema operativo y administración aplicado y el 
nivel de compromiso de los propietarios son los principales factores que impactan la adopción y crecimiento del e-commerce y su costo-beneficio sea visible y demostrado en los micronegocios del AMM.

Esto es un indicio de que aún existe el tabú de incertidumbre o desconfianza del comercio electrónico, específicamente en los microempresarios que tienen más de 45 años; sin embargo, se encuentra que los que son menores de 40 años están dispuestos a incurrir en este tipo de gastos, no solo con el afán de que sus negocios sobrevivan a la pandemia, sino también con el fin de acceder a nuevos mercados y clientes.
El entorno de evolución y el impulso de la crisis sanitaria a obligado a ciertos negocios a moverse al mundo del internet o comercio electrónico a propulsado en cierta medida capacidad de aprovechar las oportunidades de creación de valor facilitadas por el uso de la Internet. Los factores condicionantes pueden variar en el tiempo y en diferentes contextos, las microempresas deben continuar realizando actividades e iniciativas para intensificar su competitividad y progresar hacia el ecommerce. 


\section{REFERENCIAS}

Alvarado, E. (2021). Determinantes del gasto en e-commerce debido al COVID-19: un análisis empírico para los micronegocios en Monterrey, México. Paradigma Económico, 13(1), 119145. doi:10.36677/paradigmaeconomico.v13i1.14984

Bravo, S. (2011). Electronic commerce in developing countries a study of B2B E-commerce adoption by small and medium sized enterprises in Argentina. (Master of Science Thesis). INDEK School of Industrial Engineering and Management. Estocolmo, Suecia: KT

Davis FD (1989) Perceived usefulness, perceived ease of use, and user acceptance of information technology. MIS Quarterly 13(3): 319-340

Forbes Staff. (2020). México presenta plan de apoyo a pymes ante banco de desarrollo CAF. https://www.forbes.com.mx/economia-mexico-plan-apoyo-pymes-banco-desarrollo-caf/

INEGI. (2019). Instituto Nacional de Estadística y Geografía RESULTADOS DEL VALOR AGREGADO BRUTO. México: INEGI.

INEGI. (2020) Instituto Nacional de Estadística y Geografía, Encuesta Nacional de Ocupación y Empleo Nueva Edición (ENOEN) Tercer Trimestre de 2020.

Líberos, E., y Somalo, I. (2011). El libro del comercio electrónico. Madrid: Business Marketing School.

Molla, A. (2004). The impact of ereadiness on ecommerce success in developing countries: firmlevel evidence. Development Informatics. Working paper $\mathrm{N}^{\circ} 18$. Manchester, United Kingdom. Institute for Development Policy and Management University of Manchester.

OCDE (2000). Organización para la Cooperación y el Desarrollo Económicos; Pequeñas y medianas empresas: Fuerza local y alcance global.

Secretaria de Economía de México. (SE, 2020). Apoyo del Gobierno de México a las pymes en tiempos de pandemia

Tan, J., Tyler, K., \& Manica, A. (2007). Business-to-business adoption of e-commerce in China. Information \& management 44 (3), 332-351

Tavera, J. F., \& Londoño, B. E. (2014). Factores determinantes de la aceptación tecnológica del ecommerce en países emergentes. Revista Ciencias Estratégicas, 22(31), 101-119.

UNESCO. (2020). El cierre de escuelas debido a la Covid-19 en todo el mundo afectará más a las niñas.https://es.unesco.org/news/cierre-escuelas-debido-covid-19-todo-mundo-afectaramas-ninas 